\title{
Correction to: The role of proteomics in the age of immunotherapies
}

\author{
Sarah A. Hayes ${ }^{1,2} \cdot$ Stephen Clarke ${ }^{1,2,3} \cdot$ Nick Pavlakis $^{1,2,3} \cdot$ Viive M. Howell ${ }^{1,2}$
}

Published online: 29 October 2018

c) Springer Science+Business Media, LLC, part of Springer Nature 2018

\section{Correction to: Mammalian Genome}

https://doi.org/10.1007/s00335-018-9763-6

Some parts of the Abstract, Introduction and Discussion included uncited text from the following previously published chapter:

Kelly Fulton and Susan Twine (Immunoproteomics: Current Technologies and Applications in Methods Mol Biol. 2013;1061:21-57. https://doi.org/10.1007/978-1-62703 -589-7_2).

The authors apologize to the authors concerned.

The original article can be found online at https://doi.org/10.1007/ s00335-018-9763-6.

Sarah A. Hayes

sarah.hayes@sydney.edu.au

1 Bill Walsh Translational Cancer Research Laboratory, Hormones and Cancer, Kolling Institute of Medical Research, Royal North Shore Hospital, St Leonards, Sydney, Australia

2 Northern Clinical School, Faculty of Medicine and Health, University of Sydney, Sydney, Australia

3 Department of Medical Oncology, Royal North Shore Hospital, St Leonards, Sydney, Australia 